Projets

de paysage

\section{Projets de paysage}

Revue scientifique sur la conception et l'aménagement de l'espace

\section{4 | 2021}

Le paysage au prisme du politique

\title{
La Permanence, une politique de l'attention
}

Pour une compréhension située

Permanence, A Policy of Attention - In Search of a Situated Understanding

\section{Giulia Tellier et Tiphaine Hameau}

\section{(2) OpenEdition \\ 1 Journals}

Édition électronique

URL : https://journals.openedition.org/paysage/20335

DOI : $10.4000 /$ paysage. 20335

ISSN : 1969-6124

\section{Éditeur :}

École nationale supérieure du paysage de Versailles-Marseille, Institut national des sciences appliquées Centre Val de Loire - École de la nature et du paysage, École nationale supérieure d'architecture et de paysage de Bordeaux, École nationale supérieure d'architecture et de paysage de Lille, Agrocampus Angers

\section{Référence électronique}

Giulia Tellier et Tiphaine Hameau, «La Permanence, une politique de l'attention », Projets de paysage [En ligne], 24 | 2021, mis en ligne le 20 septembre 2021, consulté le 01 octobre 2021. URL : http:// journals.openedition.org/paysage/20335; DOI : https://doi.org/10.4000/paysage.20335

\section{Ce document a été généré automatiquement le 1 octobre 2021.}

La revue Projets de paysage est mise à disposition selon les termes de la Licence Creative Commons Attribution - Pas d'Utilisation Commerciale - Pas de Modification 4.0 International. 


\title{
La Permanence, une politique de l'attention
}

\author{
Pour une compréhension située \\ Permanence, A Policy of Attention - In Search of a Situated Understanding
}

\author{
Giulia Tellier et Tiphaine Hameau
}

\section{Situations}

1 Nous sommes à Morlaix, en Finistère, et nous observons une photographie aérienne de l'ancienne citadelle ouvrière de la ville où la production de cigarettes cessa en 2004 à la suite d'une succession de plans sociaux. Une bande étirée de feuillages saute aux yeux. Ce couloir de verdure est pris dans un étau de parkings, de toitures et de façades : la Manufacture des tabacs abrite, à l'arrière de ses nombreuses cours et bâtiments, des jardins. C'est entre ces murs et dans ces jardins que nous menons nos expériences de permanences, architecturale pour l'une, paysagère pour l'autre. Cette distinction est formelle ${ }^{1}$ mais elle nous permet cependant d'énoncer nos cadres de travail respectifs, car nous travaillons sur deux missions aux temporalités et maîtrises d'ouvrage différentes. Si nos cadres diffèrent, il reste un invariant de taille qui définit la permanence, cette méthode de travail que nous explorons : nous habitons le lieu du projet sur le long terme. Ainsi entre octobre 2018 et avril 2021, Giulia Tellier prend en charge la permanence architecturale du chantier du SE/cW pour l'atelier Construire. Le $\mathrm{SE} /{ }^{\circ} \mathrm{W}$ réunit la Salamandre, cinéma d'art et essai, le théâtre de l'Entresort, la troupe Catalyse, et Wart, organisateur du festival Panoramas et producteur de spectacles pour porter dans le cadre d'un marché privé la réhabilitation de près de $5000 \mathrm{~m}^{2}$ de la Manufacture en lieu culturel. De son côté Tiphaine Hameau, artiste-jardinier, prend part entre juillet 2019 et mai 2020 à la permanence urbaine tenue par Caroline Lenfant, salariée de l'atelier Construire en charge de la "Mission d'accompagnement à la stratégie de positionnement et à la programmation économique, culturelle et urbaine dans le cadre de la reconversion de la Manufacture des tabacs de Morlaix » commanditée par la communauté d'agglomération. À travers la restitution de nos deux 
expériences "habitées", nous cherchons à articuler le paysage avec le politique qui s'exprime dans chaque situation. Cette compréhension rejoint la définition de Giorgio Agamben pour qui le politique ne représente pas une discipline mais une «intensité qui tout à coup peut éclairer n'importe quel champ : l'art, la religion, l'économie, la poésie, le désir, l'amour » (Agamben, 2016).

Figure 1. Morlaix depuis son viaduc

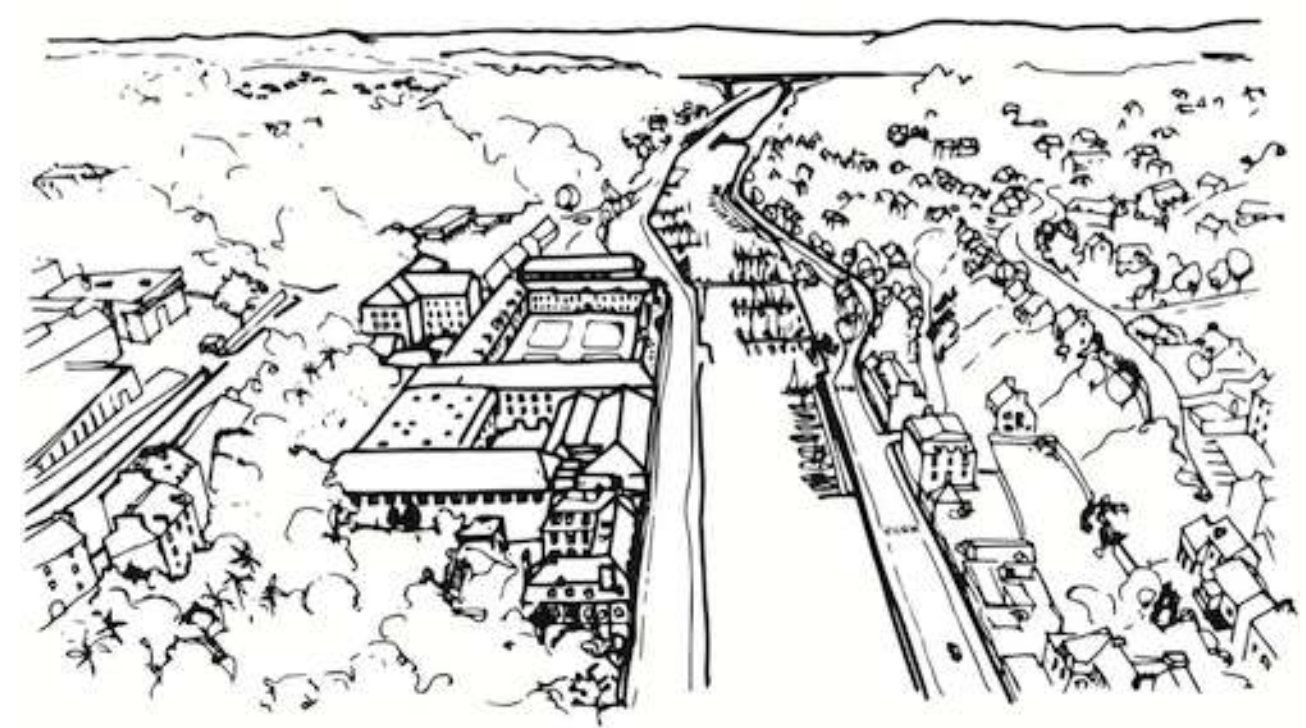

Source : Giulia Tellier.

\section{Habiter et prendre soin, une inscription politique}

Nous habitons, restons quelque part, vivons dans un lieu, tel que signifie déjà habiter vers 1050 (Rey, 1999, p. 1672). Ce lieu c'est pour la permanence de chantier une pièce principale qui est tour à tour bureau, lieu d'expositions ou de projections, avant de devenir un restaurant. Pour Tiphaine Hameau, ce lieu est constitué d'une pluralité de milieux : un verger, une friche, un ancien terrain de tennis... Que ce soit en amont ou pendant la construction, le fait d'habiter in situ permet de préparer et d'accompagner la mise en espace en partageant plutôt qu'en imposant des décisions. La temporalité longue inhérente à la méthode permet l'action quotidienne et les gestes mesurés. Pour le jardinier, faire corps avec l'espace, c'est en éprouver le temps, l'étirement du jour des premières lueurs au crépuscule, parfois durant la nuit. Une épreuve à répéter au gré des humeurs du ciel, au rythme des quatre saisons. Habiter, ici, demande donc un certain rapport au corps, une énergie à mettre dans les interactions, une connaissance des recoins et des matérialités. En rencontrant autrui, en discutant, en prêtant attention aux variations des lieux, nous tâchons de nous mettre en relation avec le paysage dans lequel nous venons nous inscrire. Le territoire, la ville, les jardins, le quartier, voire, dans le cas de la Manufacture des tabacs, l'ancien bastion industriel, deviennent ainsi un paysage existentiel selon la définition de John Brinckerhoff Jackson 
- qui «n'accomplit son identité qu'au fur et à mesure de l'existence » (Jackson, 2003, p. 116). Poussée's par la considération pour le lieu, ses passés, ses matérialités, ses habitant·e's, élu·e's, ouvrier-ère's, outils, objets, nous "ménageons » plutôt qu'aménageons. Ce ménagement est une forme douce de revendication : c'est ainsi qu'à chaque acte et instant passé sur place se fabrique du politique.

Dans les jardins, Tiphaine prend soin en remisant le plus souvent possible l'outil violent et bruyant (débroussailleuse, tronçonneuse) à la faveur du balai à gazon ou des ciseaux à herbes hautes. Il s'agit de faire "le plus possible avec et le moins possible contre " (Clément, 2006, p. 58). Sur le chantier, l'oreille est tendue aux futur.e's usagerè̀re's, mais aussi aux visiteur'e's, touristes, voisine's, associations, afin de trouver où et comment chacune peut prendre place. Au quotidien, l'attention est dirigée vers les ouvrierè̀re's : confier une clé, échanger des paroles bienveillantes, rallonger la pause du midi en prenant le temps d'une partie de pétanque. Dans le travail, nos échanges leur permettent de proposer des solutions techniques et constructives à partir de leur savoir-faire et ainsi ouvrir l'horizon de l'interprétation. Au tout exécutif se substitue le dialogue, et la hiérarchie traditionnellement à l'œuvre dans le milieu du BTP s'horizontalise. En ce sens, la permanence lutte contre l'aliénation que peut constituer le travail quand il manque de sens. Et la mise en place de petits rituels permet de désacraliser l'efficacité et de suspendre un moment les exigences de rentabilité pour entrer dans l'espace de la création et du partage : que ce soit le temps d'un café et d'un croissant, celui de la rencontre entre les comédien'ne's de Catalyse et des ouvrierè̀re's, ou encore d'un déjeuner sur l'herbe avec un guide local. Entre les murs de la Manufacture comme dans ses jardins, nous prenons le temps d'entrer en relation avec les personnes, comme avec d'autres espèces animales et végétales présentes. Mais nous devons lutter pour faire reconnaître comme travail ces moments si cruciaux que nous ne pouvons plus partager quand la fatigue et la surchage deviennent trop importantes.

4 En habitant, en devenant familierère, nous nous attachons à mettre en place des relations de confiance avec les différente's acteurs-rice's du projet. Cette question de la confiance est centrale, car, comme l'écrit Laurent Karsenty, sa construction est « l'une des dimensions permettant d'améliorer la qualité de la conduite de projet » (Karsenty et al., 2003). Par la confiance, on favorise la qualité de la communication entre les différente's acteur-rice's de la construction et de l'urbanisme, on établit un référentiel partagé, consolidant la convergence vers un objectif commun. Ce faisant, la permanence infléchit la capacité des commanditaires à s'approprier les lieux et paysages avant «livraison». En impulsant des événements spécifiques et extraordinaires tout comme de "simples" visites, il s'agit aussi d'aider le maître d'ouvrage à redéfinir, apprivoiser et exploiter les potentialités du futur lieu. Faire paysage en rassemblant les fragments constitués par les points de vue et intérêts de différentee's intervenant·e's. Par ailleurs, le soin se manifeste aussi envers les bâtiments et les lieux : vérifier les toitures et possibles entrées d'eau, la salubrité d'un plancher en bois, aérer, etc.

Notre pratique, en tant qu'élaboration et mise en partage d'un monde commun, a une teneur politique qui se fonde sur l'attention. De manière préinstitutionnelle, nous déplaçons très localement les seuils de ce qui mérite précisément cette attention et intérêt. Nous plaidons pour que les ouvrierè̀re's, aux métiers et savoir-faire souvent dévalorisés, importent. Sur un autre plan, le chant de la bergeronnette et le respect des espèces endogènes et de leur fonctionnement restent à comprendre et à valoriser 
également. Finalement il s'agit d'œuvrer à remettre au centre celles et ceux, ainsi que les savoirs et usages, qui sont d'ordinaire laissés aux marges, voire invisibilisés

Figure 2. Bureau et étagère à casques de chantier

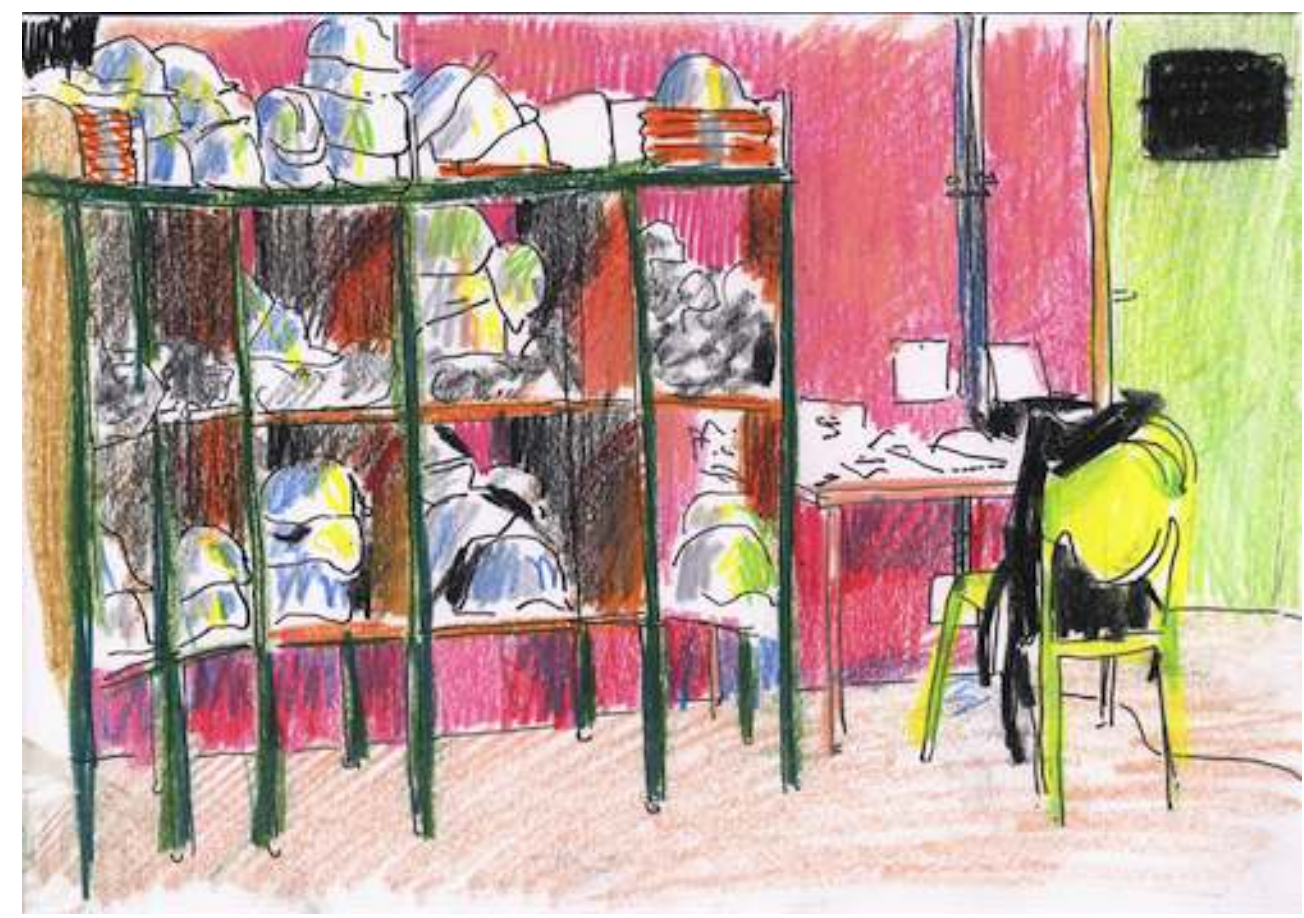

Source : Hélène Le Cam.

\section{Déplacer l'action publique?}

6 Nos terrains et notre mode d'action nous amènent aussi à rencontrer les représentants et opérateurs des politiques publiques. La Manufacture abrite aujourd'hui le siège de la collectivité territoriale, qui en est propriétaire. Dans le Cahier des clauses techniques et particulières (CCTP) qui décrit les objectifs de la mission d'étude, la communauté d'agglomération soucieuse de développer son attractivité auprès des administrée-es mais aussi des investisseurs, décrit la Manufacture comme le «futur Totem du territoire ». Sa reconversion serait inscrite « dans une dynamique de nouveau quartier tourné vers la mer, en développant les synergies entre les pôles culturel, tertiaire, économique et universitaire autour d'un projet collectif et complémentaire ». Cet extrait de discours, fédérateur et séduisant, rappelle d'autres plaquettes de projets de développement territorial. Concernant les ambitions affichées pour les jardins, la collectivité territoriale énonce " un projet de réouverture d'un jardin public, mettant en valeur les actions de la collectivité en faveur de la biodiversité ». En appeler à la biodiversité est un argument politique efficace aujourd'hui. Le plébiscite de cet atout révèle une ligne de pensée sous-jacente : la nature est ici à visée écologiste.

7 Par ailleurs la Manufacture et ses jardins représentent un enjeu de pouvoir économique : valoriser le foncier délaissé sur lequel ils prennent racine est susceptible d'accroître l'attractivité territoriale par un développement du tourisme et d'activités parallèles. Et pour une ville manquant cruellement de parcs et de jardins publics, la 
création d'un nouvel « espace vert » permet aussi de répondre à une attente sociale des administrée's.

8 Le phénomène de valorisation du foncier par le jardin peut entraîner un processus de gentrification, qui contribue lui aussi à créer une vision esthétisante de la nature en ville. La question que se pose Tiphaine Hameau, et qui oriente sa pratique et son travail, est la suivante: comment penser cette nature en ville autrement qu'à travers ces grandes lignes de pensée (écologiste ou esthétique) qui la subordonnent à des logiques productivistes, et en y intégrant une vraie considération pour le vivant dans son ensemble?

9 Les pistes sont subtiles mais révélatrices. Il s'agit par exemple de ne pas évacuer la matière végétale mais plutôt la réutiliser sur place, l'intégrer au projet pour structurer sur le temps long le lieu et les usages. Petit à petit, cela fonctionne et convainc: si à l'issue de la mission d'étude toute décision concernant les occupations et reconversions des espaces clos, couverts, et vacants semble suspendue, les jardins ont fait l'objet d'un accord spécifique pour que le travail entrepris par Tiphaine Hameau puisse se poursuivre en 2021.

\section{Révéler les enjeux, faire avec les frictions}

Pour identifier les enjeux et frictions qui se cristallisent dans les possibles futurs de la Manufacture (occupations d'espaces et nombre de mètres carrés attribués aux différentee-s acteur-rice's, qu'ileelle's soient déjà présentees dans la Manufacture ou «candidate's» à s'y établir, modes de gouvernance, rentabilité économique ou communicationnelle, etc.), nous devons discerner depuis quelle position et dans quelles conditions les discours sont produits. Nos propres mots et discours depuis nos positions de "tiers personnage", doivent être mesurés et suivre le système de valeurs auquel notre pratique professionnelle nous affilie. Nous permettons ainsi le passage des messages entre des milieux très divers et entre des intérêts parfois contradictoires. Dans son travail Olivier Chadoin énonce cette nécessitê de "passage du langage d'un univers spécialisé à un autre» (Chadoin, 2001). Cette "traduction» permet de faire face à la complexité des processus de conception tout en réduisant les incertitudes dues aux différentes interprétations d'un même message. Après tout on ne raconte pas à des enfants en maternelle comme on le fait à une élue politique ou à un groupe de personnes en formation d'électricité.

11 Parmi nos rencontres, nous comptons le voisinage de la Manufacture. Propriétaires d'une partie de l'ancienne maison du directeur et de ses jardins, salariée-es travaillant au siège de direction d'un journal local ou des services de l'agglomération, locataires d'appartements, utilisateur.rice's de l'école de danse ou des cabinets de kinésithérapeutes, pour toutes ces personnes, les jardins ont représenté durant l'étude un objet de projections très divergentes. Certain-e-s, effrayée's du possible vis-à-vis avec leurs jardins privés, souhaitaient voir une partie des jardins condamnée. D'autres y voyaient l'opportunité d'y mener des concerts et activités culturelles de plein air, et d'autres encore d'en faire un laboratoire de plantes aux propriétés anti-cancéreuses. D'aucuns'es y voyaient l'opportunité simple et appréciable d'y promener leurs enfants en bas âge sans devoir s'inquiéter de la proximité de véhicules. Nous voyons là combien un paysage se constitue d'une multiplicité d'images, de projections et de représentations mais cela tient aussi au caractère clos et encore enfriché des jardins, 
qui leur confère un potentiel de "médiation ", d'ouverture de voix et de voies. Cette idée de multiplicité constitue pour certain'es la base matérielle du paysage (Carrozza, 2009). Cependant, une fois réunis ces souhaits, paroles, témoignages et idées relatives à la gestion des lieux, le défi qui se pose est: comment s'en faire le relais ? Comment défendre une organisation, une gestion des lieux la plus commune et horizontale possible? Par ailleurs, chercher à rassembler les souhaits, à y répondre, n'est-ce pas déjà neutraliser le conflit et son potentiel d'invention en faveur du lissage opéré par la puissance économique et administrative? Si la permanence constitue un outil " alternatif » censé s'écarter des procédures top-down établies «au sein de cénacles fermés réunissant les seuls grands élus, experts et représentants du secteur privé » (Béal et Rousseau, 2014), on sait combien cet alternatif peut n'être qu'une façade, un outil rhétorique, argument consensuel ou marketing de fabrication de projet. Dans le cadre de l'étude urbaine, les membres de la permanence ont souvent été frappés par la vision très prédéfinie et nette qu'avaient certain'e's élu·e's ou technicien.ne-s quant au projet et aux jardins : le paysage commun était déjà constitué selon un certain prisme.

12 À cet égard, la délégation horizontale du pouvoir décisionnel et de mise en action, considérée comme constitutive de la permanence par Édith Hallauer, ne se faisait que difficilement dans le cadre de l'étude urbaine (Hallauer, 2015). Nous nous sommes interrogée's sur les motivations d'un tel contrôle et sur cet attachement à la hiérarchie verticalisée, et nous avons conçu une hypothèse: pendant ces permanences, nous avions les clés et avions ouvert des portes. Cette relation exclusive aux lieux et la gestion presque "domestique » que nous en faisions ont peut-être amené certains acteurs décisionnels à se sentir dépossédés du projet, et donc à recadrer la méthode de manière administrativement « acceptable ».

Figure 3. Visite de chantier

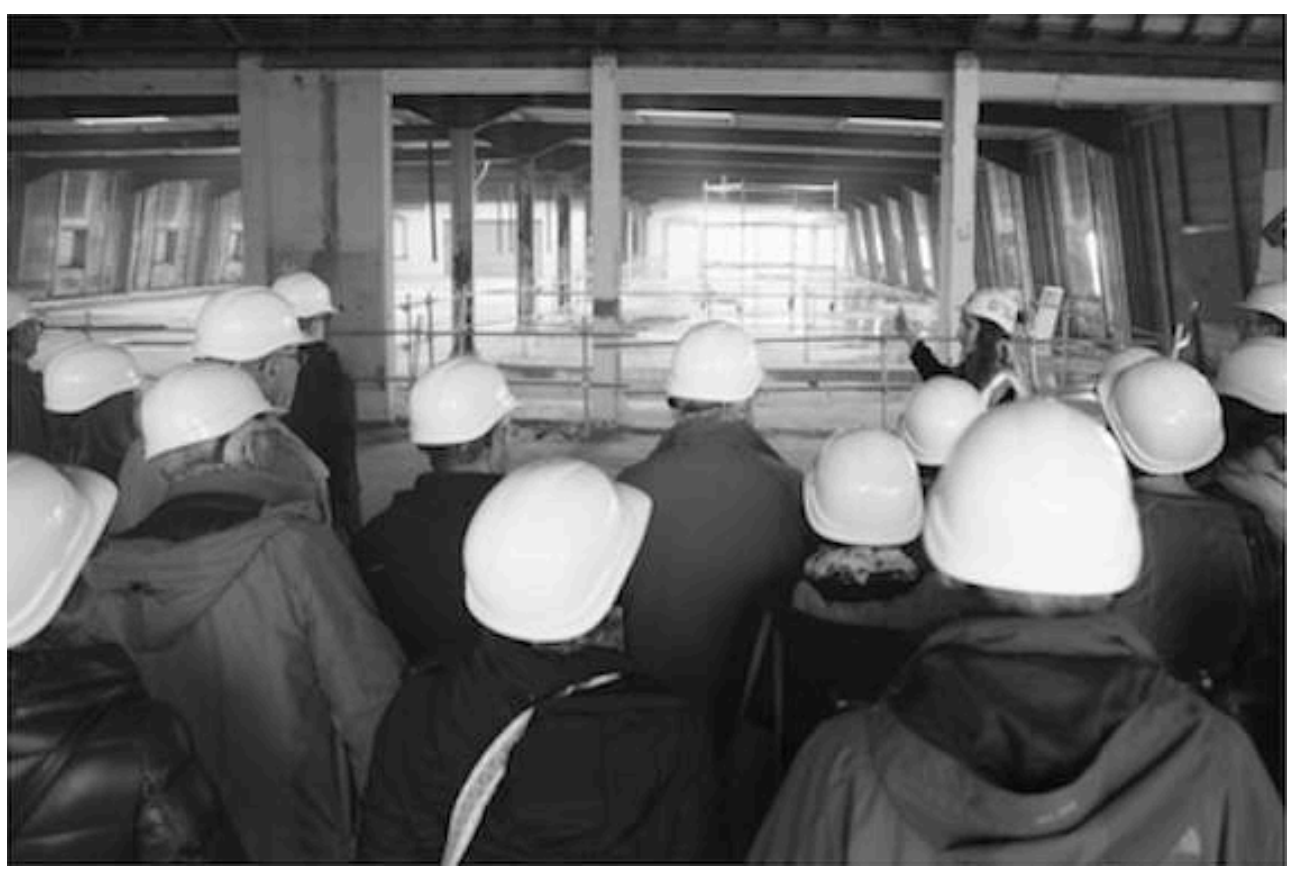

Source : Lisa Zewuster. 


\section{Relations à l'œuvre et au vivant} fabriqués et tissés ponctuellement entre riverain·e's et élue·s, entre maîtrise d'œuvre et technicien'ne's, si déconnectée's parfois du fait des lourdeurs administratives, en sont la preuve. Mais à notre sens ces liens ne peuvent s'implémenter sans la mise en route d'un processus de "déprise d'œuvre », tel que théorisé par Édith Hallauer. En termes marxistes, nous considérons que nous participons à la production collective d'un objet (projet, chantier) et que la source située et individuelle n'est tout simplement pas prépondérante. Par ailleurs, si le paysage est à fois œuvre d'un «travail » volontaire et spécifique, il est aussi le fruit du travail des usagerère-s, personnes privées ou publiques, et enfin l'œuvre de celles et ceux les moins ouvertement interventionnistes que sont les visiteurs, promeneurs, spectateurs. Ces derniers produisent par leurs usages, attentes, sensibilités, manières de parler, une façon d'habiter le paysage et de le faire qui est à la fois in visu, in situ, in coropore, et in spirito. Par ailleurs offrir l'accès et le partage des jardins de la Manufacture c'est ouvrir un espace déjà habité d'espèces vivantes autres que la nôtre. Or, cette ouverture nécessite de penser aux espèces endogènes, dont l'habitat est susceptible d'être détérioré par l'aménagement humain, ses usages, ses circulations.

15 À l'image des conceptions développées par Baptiste Morizot (Morizot, 2020), nous tâchons de considérer le vivant et le paysage non plus comme un décor ou un support, ni une réserve de ressources à disposition mais plutôt comme le lieu et le tissu de relations qui possèdent leur propre "consistance ontologique ». Ainsi Tiphaine raconte-t-il les plantes, les nomme, ou encore nous informe sur les chants et bruissements d'oiseaux entendus dans les jardins. Ces chants et sons ne forment plus alors un "bruit blanc» mais un langage racontant quelque chose de singulier sur le paysage partagé. À cet égard, Tiphaine questionne aussi la gestion des plantes invasives orchestrée par Morlaix Communauté, en faisant sienne la formule de Jean-Pierre Le Dantec : « décider, parmi nos végétaux communs, ceux dont l'origine est indigène (pour ne pas dire "de souche") est une gageure» (Le Dantec, 2011, p. 127). Au-delà du jugement restrictif, voire moral, de ce qui relève ou non de l'endogénéité, et de la gestion de la prolifération d'une espèce végétale, nous considérons qu'il est utile de remettre en discussion critique les rhétoriques du local et de la "source». En effet ces dernières contribuent parfois à figer les identités affiliées aux lieux et aux paysages. (Clément, 1999, p. 60). 
$16 \mathrm{Au}$ travers de nos permanences nous avons tenté de transmettre ces réflexions et sensibilités aux milieux, qui constituent les prémices d'une recherche. Comment modifier nos manières de nous arroger des droits, de projeter des usages et d'extraire les richesses d'un milieu pour notre unique profit? L'enjeu politique du paysage est bien là : rendre sensible ce quelque chose à voir, à lire, à partager, autre que ce que nous nous contentons d'ordinaire de voir. Il existe, dans nos milieux, autour de nous, des significations riches à traduire et à comprendre, tant sur le plan humain et social que venant d'autres espèces avec qui nous cohabitons.

\section{Conclusion}

17 Si les ouvertures du chantier et des jardins ont permis aux " publics » de s'habituer à la fréquentation des lieux ainsi que la création de liens inédits, cela n'est pas toujours advenu en une "fusion paisible des différences " (Rancière, 2020, p. 65). En effet la permanence fabrique aussi parfois du conflit, qui reste nécessaire pour s'ouvrir aux raisons et motivations d'autrui. Le conflit permet également et paradoxalement de comprendre le réseau de dépendances mutuelles qui préside à la fabrication du commun tout en échappant à la logique binaire de camps qui s'affrontent.

La pratique de la permanence entrelace étroitement les domaines professionnels et privés. Elle constitue également une manière d'interroger l'énigme politique par excellence qu'est la vie en commun dans un monde d'altérités. Le choix de cette façon d'être au monde permet de développer un type d'attention qui est de l'ordre de la disponibilité aux pluralités du vivant. Au droit de regard et à la fabrication du paysage par l'œil humain, nous appliquons une suspension, un retrait. À notre avis, intégrer à la fabrication des paysages les milieux et les autres espèces, c'est chercher à dépasser un anthropocentrisme qui, dans nos métiers et façons d'entrer en relation, n'est que rarement remis en question. Voilà un horizon de réflexion et de pratique qu'il nous semble intéressant de proposer.

La permanence telle que nous avons tâché de la mettre en œuvre vise à produire une nouvelle forme d'intégration : rassembler le dedans et le dehors, le présent et le passé, l'intime et le collectif. C'est une invitation à faire ou refaire un monde en se positionnant et en invitant autrui à se positionner comme sujet politique et poétique.

Merci à Caroline Lenfant sans qui cet article n'aurait pu voir le jour.

\section{BIBLIOGRAPHIE}

Agamben, G., " Credo nel legame tra filosofia e poesia. Ho sempre amato la verità e la parola ", entretien avec Antonio Gnoli, La Repubblica, 15 mai 2016.

Béal, V. et Rousseau, M., « Alterpolitiques ! », Métropoles, n 15, décembre 2014, URL : http:// journals.openedition.org/metropoles/4948; DOI : https://doi.org/10.4000/metropoles.4948 
Carrozza, M. L., « Paysage urbain : matérialité et représentation », Cahiers du Centre de recherches historiques, $\mathrm{n}^{\circ} 17,1996$, mis en ligne en février 2009, URL : http://journals.openedition.org/ccrh/ 2600; DOI : https://doi.org/10.4000/ccrh.2600

Castells, M., La Question urbaine (1972), Paris, Maspero, 1981, p. 26-27.

Chadoin, 0. , « De la décision à sa traduction : fidélité, re-création et bricolage intellectuel ", Espaces et Société. Projet urbain, maîtrise d'ouvrage, commande, $n^{\circ}$ 2-3, 2001.

Clément, G., Une écologie humaniste, Paris, Aubanel, 2006, p. 58.

Clément, G., Le Jardin planétaire, Paris, Albin Michel, 1999, p. 60.

Dardot, P. et Laval, C., Commun. Essai sur la révolution au XXI e siècle, Paris, La Découverte, 2015.

Habermas, J., L'Espace public (1962), Paris, Payot, 1988.

Hallauer, E., « Habiter en construisant, construire en habitant : la "permanence architecturale", outil de développement urbain ?", Métropoles, nº 17, 2015, décembre 2015, URL : http:// journals.openedition.org/metropoles/5185; DOI : https://doi.org/10.4000/metropoles.5185

Jackson, J. B., À la découverte du paysage vernaculaire (1984), Arles, Actes Sud, 2003, p 116

Karsenty, L., Barcellini, F. et Grosse, C., « Quelle démarche pour favoriser la construction de relation de confiance dans un projet de conception? ", dans Karsenty, L. (dir), La Confiance au travail, Toulouse, Octares, 2013, p. 187-207.

Le Dantec, J.-P., Poétique des jardins, Arles, Actes Sud, 2011, p. 127.

Morizot, B., Manières d'être vivant, Arles, Actes Sud, 2020.

Rancière, J., Le Temps du paysage, Paris, La Fabrique, 2020, p. 65.

Rey, A., Dictionnaire historique de la langue française, t. 2, Paris, Le Robert, 1999, p. 1672

Ricard, S., « La permanence pour un urbanisme vivrier », conférence diffusée en direct le 27 octobre 2020, URL : https://www.youtube.com/watch ?v =ozc8lKGqndU

Sansot, P., Jardins publics, Paris, Payot, 2003, p. 66.

Schama, S., Le paysage et la Mémoire (1995), Paris, Seuil, 1999.

\section{NOTES}

1. Nous considérons l'architecture comme partie intégrante d'un paysage, en tant que «tout paysage naît de la rencontre d'un lieu sensible et d'un être sentant » (Sansot, 2003, p. 66).

\section{RÉSUMÉS}

Cette contribution est issue d'un partage entre une architecte et un artiste-jardinier, attaché'e-s à l'exercice d'écriture comme outil de mise en perspective de réalités vécues et moyen de restitution de leurs expériences. Elle offre le témoignage d'une expérience commune, celle 
d'«habiter " un lieu, le temps d'un chantier de réhabilitation et d'une étude urbaine et paysagère, avec une même posture patiente qui conduit de facto à (a)ménager autrement. Le texte qui suit décrit une méthode de travail connue sous le nom de "permanence", en la problématisant, en reliant les actes et les relations nouées lors de ces vécus à différentes échelles de compréhension du politique. Nous cherchons à extraire de ces situations des données indicatives sur nos manières d'être au monde, d'agir et de travailler collectivement, au sein de paysages par essence morcelés, fruit de regards, de mains et d'interrelations procédant d'origines, d'âges, voire d'idéologies très divers.

This contribution is the outcome of exchanges between an architect and a garden-artist, both of whom are attached to the act of writing as a means of putting real life situations into perspective and providing feedback on their own experiences. It is the account of a shared experience, that of "inhabiting" a site during a rehabilitation project and an urban landscape development study, with the same patient posture which leads de facto to a different way of approaching the project. The article describes a working method, referred to as 'permanence', by problematising it ; by linking the acts and relationships established during these experiences to different scales of understanding of policy. We seek to extract from these situations information indicative of our ways of being in the world, of acting and working collectively within landscapes that are essentially fragmented and which are the result of views, actions, and interrelationships stemming from origins, ages, and even ideologies that are extremely diverse.

\section{INDEX}

Mots-clés : jardins, patrimoine, méthodes de projet, habiter, propriété

Keywords : gardens, heritage, project methods, inhabit, property

\section{AUTEURS}

\section{GIULIA TELLIER}

Giulia Tellier est architecte HMONP, diplômée en 2018 avec mention recherche, salariée de l'atelier Construire. En 2020 elle cofonde le Groupe de recherche et d'action sur la production de l'espace (GRAPE, https://grape.noblogs.org/). giulia.tellier[at]gmail[dot]com

\section{TIPHAINE HAMEAU}

Tiphaine Hameau, artiste-jardinier autodidacte, réalise des jardins comme si c'était le sien, celui qu'il n'a pas encore. Il prévoit de s'arrêter là où il l'aura trouvé. 